\title{
Prevalence of Hepatitis B and Knowledge About Hepatitis B Among Migrant Workers in Shandong Province, China: A Cross-Sectional Study
}

\author{
Yan Yang ${ }^{1, *} ;$ Ming Yan ${ }^{2}$; Meng Yue ${ }^{3}$; Xiaohua Wang ${ }^{4}$; Wei Zhang ${ }^{1}$; Jie Li ${ }^{1}$; Shuqing Li $^{1}$ \\ ${ }^{1}$ Health examination Center, QiLu Hospital, Shandong University, Jinan, China \\ 2 Department of Geriatric Gastroenterology, QiLu Hospital, Shandong University, Jinan, China \\ ${ }_{3}^{3}$ Department of Gastroenterology, Jinan Central Hospital, Shandong University, Jinan, China \\ 4 Jinan infectious Disease Hospital, Jinan, China \\ ${ }^{*}$ Corresponding Author: Yan Yang, Health examination Center, QiLu Hospital, Shandong University, P. O. Box: 250012, Jinan, China. Tel: +86-53182166920, Fax: +86-53182166921, E-mail: \\ yangyan1021@tom.com
}

Received: January 6, 2015; Revised:January 25, 2015; Accepted: February 16, 2015

\begin{abstract}
Background: China is a country with a high prevalence of hepatitis B. As a special population, migrant workers are more vulnerable to hepatitis B.

Objectives: The present study was conducted to gain insight into the prevalence of hepatitis B and knowledge about hepatitis B among migrant workers in Shandong Province, China, as well as to explore a series of strategies for preventing and controlling the spread and prevalence of hepatitis B.

Patients and Methods: A cross-sectional study was conducted without age restriction, in Shandong Province in China. The sample-size was determined scientifically. The study population was selected using the random multistage cluster sampling. Personal information, including sex, age, ethnicity, marital status, education level, years of duration of stay in Jinan and health insurance were obtained from 2065 migrant workers. Moreover, blood samples were collected for hepatitis B infection screening. Knowledge about hepatitis B was assessed using a self-administered questionnaire. Correct response rates were calculated.

Results: Of 2065 migrant workers tested for Hepatitis B Surface Antigen (HBsAg), 167 (126 men and 41 women) tested positive for HBsAg; the overall prevalence was 8.1\%. The prevalence rates in men and women were $9.6 \%$ and $5.4 \%$, respectively. The prevalence rates in subjects 18 - 30 years old and 30 years older were $3.5 \%$ and $9.3 \%$, respectively. There were statistically significant differences between those groups (P $<0.05$ ). Correct response rates for the transmission of hepatitis B questions were low, especially for a question regarding whether hepatitis $\mathrm{B}$ can be transferred by sexual contact (36.8\%) and whether it can be transferred from mothers to infants (33.9\%). A total of $80.9 \%$ of migrant workers correctly responded that vaccination is effective for hepatitis B. However, $68 \%$ of migrants also had the misconception that it is necessary to receive a booster vaccination.

Conclusions: The hepatitis B virus infection rate in migrant workers is higher than average infection rates in China, and these workers' knowledge regarding hepatitis B is poor. It is urgent that an appropriate program be undertaken for the prevention and control of hepatitis B among migrant workers.
\end{abstract}

Keywords: Transients and Migrants; Hepatitis B; Knowledge; Cross-Sectional Studies; Questionnaires

\section{Background}

Since the beginning of economic reform, China's market economy has expanded, with rapid modernization and industrialization resulting in massive internal ruralto-urban migration within China (1). The number of migrant workers increased from 50 million in 1990 to 121 million in 2000 (2). According to official estimates, there are approximately 220 million migrant laborers in China (3). Hepatitis B is a major worldwide public health problem (4). As the most serious type of viral hepatitis, the interactions between the Hepatitis B Virus (HBV) and the host immune system is responsible for approximately one million deaths due to liver failure and cirrhosis (5, 6 ), and more than $75 \%$ of the hepatocellular carcinomas worldwide every years develop from HBV infections (7). It is estimated that more than two billion people have been infected with HBV worldwide, one-third of whom are in
China. China is thus a country with a high prevalence of hepatitis B. According to the fourth national socio-epidemiological survey, the prevalence of hepatitis B surface antigen among Chinese people younger than 60 years old was $7.20 \%$ in 2006 (8). Over the last 20 years, a vaccination program has contributed to a reduction in HBV infection, decreasing from nearly $10 \%$ to approximately $7 \%$ in the general population (9). However, hepatitis B remains a serious challenge for disease control in China, and the prevalence of hepatitis B among migrant workers is not optimistic. Migrant workers comprise a distinct and underprivileged group in urban China. They have left their homes and live in overcrowded accommodations with poor sanitation, and they are not entitled to certain rights, including open employment opportunities, free education, social welfare and medical benefits. Further-

Copyright ( 2015, Iranian Red Crescent Medical Journal. This is an open-access article distributed under the terms of the Creative Commons Attribution-NonCommercial 4.0 International License (http://creativecommons.org/licenses/by-nc/4.0/) which permits copy and redistribute the material just in noncommercial usages, provided the original work is properly cited. 
more, they only take jobs that city residents reject, such as handling of corpses, sewage and chemical waste treatment, and construction work. Therefore, migrants have a high risk of HBV infection. As reported in many studies in China, migrant workers have made a greater contribution to maintaining a higher hepatitis B incidence than local residents (10).

It is therefore very necessary to provide knowledge about the hepatitis B epidemic status of migrant workers, and it is equally necessary to undertake an appropriate program for the prevention and control of hepatitis $B$ among migrant workers.

\section{Objectives}

The purpose of study was to gain insight into the prevalence of hepatitis B and knowledge about hepatitis B among migrant workers in Shandong Province, China.

\section{Patients and Methods}

\subsection{Ethics Statement}

This cross-sectional descriptive study was performed on 2065 migrant workers in Jinan City, the capital of Shandong Province, from December 2013 to April 2014. The consent protocol and the procedure were approved by the Ethics Committee of Qilu Hospital of Shandong University in China. Prior to enrollment in the study, the research team members informed the participants regarding the inclusion criteria and the research protocol. Participation in the study was completely voluntary, and the participants had the option of leaving the entire questionnaire blank if they did not wish to participate. The survey data was presented in an aggregated manner. They were assured of the confidentiality of their responses. All these policies were also printed in the front cover of the questionnaire. An informed written consent was obtained from all of the participants. The data were analyzed anonymously.

\subsection{Design and Participants}

Shandong Province, the second largest province in China, has experienced rapid economic growth and has attracted a large migrant population. By the end of 2012, Shandong had approximately 6.91 million migrants. The study that we report on here was conducted in Jinan, the capital of Shandong Province.

The survey sample was randomly selected using a cluster sampling approach. The sample-size was determined using the statistical cluster-sampling technique according to the formula: $n=p(1-p) N^{2} / N^{2}+Z^{2} p(1-p)$. The participants were randomly recruited from several occupational clusters: the construction industry; mining; manufacturing; marketing; restaurants and entertainment; and commercial services. A list of migrant workers from above clusters was obtained using a registration book. The inclusion criteria for the study subjects were: 1) "migrants worker" was defined in the study as a person who was 18 years old or older, possessed a legal rural hukou (formally registered permanent residents in a rural area in China), and had been granted the legal right to work temporarily in urban for at least six months; 2 ) either sex;3) any socioeconomic level; 4) voluntarily agreed to participate, including a blood test; and 5) capable of independent communication and providing informed consent for this study. All of the participants were eventually informed of their blood test results. In total, 2065 migrant workers were enrolled.

\subsection{Demographic Characteristics}

In total, 2065 valid questionnaires and blood samples were obtained. The valid sample comprised 1310 male and 755 female subjects. We obtained the characteristics of the participants using a self-administered questionnaire. Personal information, including sex, age, ethnicity, marital status, education level, years of duration of stay in Jinan and health insurance, were collected. Educational level was classified into five levels: illiterate (i.e. people who could not read newspapers or magazines or write a short note); elementary school (i.e. people who attended up to 6 years of schooling or people without schooling who were not illiterate); junior high school (i.e. schooling for 7 - 9 years); senior high school (i.e. schooling for 10 - 12 years); and college, university or higher (i.e. complete or incomplete postsecondary school). Health insurance status was categorized as insured or uninsured. A person was defined as insured if he or she was covered by the New Rural Cooperative Medical Scheme (NRCMS) or by private insurance.

\subsection{Laboratory Testing}

Blood samples were collected and tested for Hepatitis B Surface Antigen (HBsAg) using electrochemiluminescence (Architect, Abbott Laboratories, and Abbott Park, IL, USA), according to the manufacturer's instructions. The interpretation of hepatitis B serologic test results was defined according to the official Web site of the United States Centers for Disease Control and Prevention (CDC) (11). The HBsAg positivity was considered indicative of current HBV infection. Those who tested negative for all HBV serological markers were considered to be nonimmune and noninfected.

\subsection{Knowledge Responses Regarding Hepatitis $B$}

Knowledge regarding hepatitis B was assessed using a self-administered questionnaire consisting of 22 questions, including etiology, symptoms, transmission, and vaccination. Respondents were read the following statement: "Have you ever heard of a disease called hepatitis B? Is hepatitis B an infectious disease caused by the hepatitis B virus? Is China a country with a high prevalence of hepatitis B? "They were then asked if they thought hepatitis B can 
Yang Yet al.

be spread during sexual intercourse, during childbirth, by sharing razors, and by someone who looks healthy. Specifically, we asked whether they thought hepatitis B can be prevented and whether vaccination is effective for hepatitis B. Finally, we asked each respondent whether hepatitis B disease causes liver failure, cirrhosis and liver cancer. Each response to the questions was scored as "yes" or "no". Correct response rates were calculated.

\subsection{Data Collection}

Doctors and nurses, after unified training at our center, performed the survey. Under the investigators' guidance, the respondents completed the questionnaire themselves. For quality control, the completeness of the ques- tionnaire was checked before the survey was over. If some information was missing from the survey, the individuals were resurveyed.

\subsection{Statistical Analysis of Results}

The respondents' demographic characteristics, positivity for HBsAg, and correct response rates to the questions were described using proportions. The SPSS software program, version 10, was used for all of the statistical analyses. Ninety-five percent confidence intervals (CIs) for the proportion of individuals who were HBsAg-positive were calculated for each variable. Differences among the groups were assessed, and p-values of less than 0.05 were considered to be statistically significant.

Table 1. Demographic Characteristics of Respondents and Incidence of Positivity for Hepatitis B Surface Antigen ${ }^{\text {a }}$

\begin{tabular}{|c|c|c|c|c|}
\hline Characteristics & Sample Size & HBsAg Positive ${ }^{b}$ & CI, 95\% & P Value \\
\hline Gender & & & & 0.03 \\
\hline Male & 1310 & $126(9.6)$ & $(8.0-11.2)$ & \\
\hline Female & 755 & $41(5.4)$ & $(3.8-7.0)$ & \\
\hline Total & 2065 & $167(8.1)$ & $(6.9-9.3)$ & \\
\hline Ethnicity & & & & 0.21 \\
\hline Minority Chinese & 46 & $4(8.7)$ & $(3-21)$ & \\
\hline Han Chinese & 2019 & $163(8.1)$ & $(6.9-9.3)$ & \\
\hline Marital status & & & & 0.17 \\
\hline Married & 1520 & $122(8.0)$ & $(6.7-9.4)$ & \\
\hline Previously married & 36 & $3(8.3)$ & $(2-22)$ & \\
\hline Unmarried & 545 & $42(7.7)$ & $(5.5-9.9)$ & \\
\hline Age group,y & & & & 0.048 \\
\hline $18-30$ & 435 & $15(3.5)$ & $(1.8-5.2)$ & \\
\hline $30-40$ & 805 & $65(8.1)$ & $(6.9-9.3)$ & \\
\hline $40-50$ & 610 & $66(10.8)$ & $(8.3-13.3)$ & \\
\hline $50-66$ & 215 & $21(9.8)$ & $(5.8-13.8)$ & \\
\hline Education & & & & 0.31 \\
\hline Illiterate & 46 & $4(8.7)$ & $(3-21)$ & \\
\hline Elementary school & 635 & $45(7.1)$ & $(5.1-9.1)$ & \\
\hline Junior high school & 1045 & $91(8.7)$ & $(7.0-10.4)$ & \\
\hline Senior high school & 297 & $24(8.1)$ & $(6.9-9.3)$ & \\
\hline College or above & 42 & $3(7.1)$ & $(2-20)$ & \\
\hline Insurance coverage & & & & 0.13 \\
\hline The New Rural Cooperative Medical Scheme & 1100 & $94(8.5)$ & $(6.9-10.1)$ & \\
\hline Private insurance & 37 & $3(8.1)$ & $(2-22)$ & \\
\hline Uninsured & 928 & $70(7.5)$ & $(5.8-9.2)$ & \\
\hline Duration of stay in Jinan, $y$ & & & & 0.09 \\
\hline Less than 1 & 588 & $43(7.3)$ & $(5.2-9.4)$ & \\
\hline $1-5$ & 1021 & $91(8.9)$ & $(7.2-10.6)$ & \\
\hline More than 5 & 456 & $33(7.2)$ & $(4.8-9.6)$ & \\
\hline
\end{tabular}

a Abbreviations: CI, confidence interval and HBsAg, Hepatitis B Surface Antigen.

b Values are presented as No. (\%). 
Yang Yet al.

\section{Results}

\subsection{Demographic Characteristics and Positive HB- sAg Among Migrant Workers}

The demographic characteristics and positivity for HBsAg among the respondents are presented in Table 1. A total of 2087 questionnaires were distributed, and 2065 valid questionnaires and blood samples were returned; the survey response rate was $98.9 \%$. The participants ranged in age from 18 to 66 years old, and the average age in the sample was 38.5 years old $(S D=2.9)$. Most of the respondents were married (73.6\%), and more than 63\% were male (1310 men); $89 \%$ were between 18 and 50 years of age, and $87 \%$ had junior high school educations or lower. The duration of residence in Jinan was broadly distributed: $28.5 \%$ (588/2065) had lived there less than 1 years, $46.4 \%$ (1021/2065) for 1 to 5 years, and 22.1\% (456/2065) for more than 5 years. Forty-five percent of the respondents did not have any kind of health insurance. Of the 2065 migrant workers tested for HBsAg, 167 (126 male and 41 female subjects) tested positive for HBsAg; the overall positivity was $8.1 \%$. (95\% CI: $6.9 \%$ - 9.3\%). The prevalence in male and female subjects was 9.6\% (95\% CI: 8.0\%-11.2\%) and 5.4\% (95\% CI: $3.8 \%-7.0 \%)$, respectively. The prevalence in subjects 18 - 30 years old and 30 years old or older was 3.5\% (95\%
CI: 1.8\% - 5.2\%) and 9.3\% (95\% CI: 7.9\% - 10.7\%), respectively. There were statistically significant differences between the groups $(\mathrm{P}<0.05)$ ( $\mathrm{P}$ values were listed in Table 1$)$. Chi-square tests were used to compare the demographic characteristics of the respondents. Table 1 provides our findings from these bivariate comparisons. Positive for HBsAg was significantly associated with sex and age.

\subsection{Assessment of Hepatitis B Knowledge}

Table 2 describes the current status of knowledge among migrant workers. The majority of survey respondents had heard of a disease called hepatitis B (89.8\%), but only a minority knew that China is a country with a high prevalence of hepatitis B (38.9\%). Correct response rates for questions on the transmission of hepatitis B were low, especially the question of whether hepatitis B can be transferred by unsafe sexual contact (36.8\%) and whether it can be transferred from mother to infant (33.9\%). The proportions who knew HBV can be prevented by using condoms during sex was $42.9 \%$, a total of 80.9 percent of migrant workers knew that vaccination is effective for hepatitis B. However, $68 \%$ of migrants also had the misconception that it is necessary to receive a booster vaccination. In addition, only $26.9 \%$ knew that hepatitis B disease can causes liver failure, cirrhosis and liver cancer.

\begin{tabular}{|c|c|c|}
\hline Questions & $\begin{array}{c}\text { Correct } \\
\text { Response }\end{array}$ & $\begin{array}{c}\text { Correct Re- } \\
\text { sponse Rates, } \%\end{array}$ \\
\hline Have you ever heard of a disease called hepatitis B? & 1853 & 89.7 \\
\hline Is hepatitis B an infectious disease caused by the hepatitis B virus? & 210 & 50.8 \\
\hline Is China a country with high prevalence of hepatitis B? & 803 & 38.9 \\
\hline Can hepatitis B be transmitted by unsterilized needles, syringes and surgical instruments? & 845 & 40.9 \\
\hline $\begin{array}{l}\text { Can a person be infected with hepatitis B by injecting blood or blood products contami- } \\
\text { nated by hepatitis B virus? }\end{array}$ & 925 & 44.8 \\
\hline Can hepatitis B be transferred by barbers or through ear and nose piercing? & 785 & 38 \\
\hline Can hepatitis B be transmitted by sexual conduct? & 760 & 36.8 \\
\hline Can hepatitis B be transmitted from mother to infant? & 700 & 33.9 \\
\hline Can hepatitis B be transmitted from one person to another in the family? & 1135 & 55 \\
\hline $\begin{array}{l}\text { Can hepatitis B be transmitted by contaminated water/food prepared by person suffering } \\
\text { from hepatitis B? }\end{array}$ & 1255 & 60.8 \\
\hline Can a person be infected with hepatitis $B$ if they are bitten by a mosquito? & 1380 & 66.8 \\
\hline Can hepatitis B be prevented? & 1285 & 62.2 \\
\hline Is vaccination effective for hepatitis $B$ ? & 1670 & 80.9 \\
\hline Are the antibodies after vaccination permanent? & 755 & 36.6 \\
\hline Is it necessary to receive a booster vaccination? & 660 & 32 \\
\hline Can using condoms during sexprevent hepatitis B? & 885 & 42.9 \\
\hline Should hepatitis B testing be performed before marriage? & 995 & 48.2 \\
\hline Can a person with hepatitis B be infected for life? & 1125 & 54.5 \\
\hline Can someone with HBV infection look like a healthy person? & 1010 & 48.9 \\
\hline The early symptoms of hepatitis B are fever, running nose, cough, etc. & 475 & 23 \\
\hline Is there any miracle drug to treat hepatitis $B$ ? & 1195 & 57.9 \\
\hline Can hepatitis B cause liver failure, cirrhosis and liver cancer? & 555 & 26.9 \\
\hline
\end{tabular}




\section{Discussion}

Though in different regions of China there is different HBsAg positivity rate, an epidemiological study among the general population in Shandong revealed that the HBsAg prevalence among participants younger than 60 years of age was $3.39 \%$ in 2009 (12). We found that the rate of serologic testing for hepatitis B migrant workers in the current study was $8.1 \%$, much higher than that of general population in Shandong. Shandong Province is an affluent province; however, the high migrant workers may negatively influence the prevalence of HBsAg.

The following factors might explain the high prevalence of hepatitis B among migrant workers: 1) Most migrant workers live in overcrowded and poor sanitary conditions; furthermore their diets are simple; in a word, their quality of life is poor; 2) Because of work overloads, longterm depression and stress, migrant workers have a high risk of damaging the human biological clock rhythm, causing a decrease in resistance; 3) Health knowledge related to hepatitis $B$, such as the source of the infection and modes of transmission, in a susceptible population such as migrant workers is poor; and 4) The mobility and instability of their occupations cause migrant workers to have more opportunities for contact with hepatitis B carriers.

Hepatitis B virus testing would be associated with certain demographic characteristics. In this study, we found a strong relationship between positive HBsAg with sex and age. The prevalence rates in male and female subjects was 9.6\% (95\% CI: 8.0\% - 11.2\%) and 5.4\% (95\% CI: 3.8\% - 7.0\%), respectively. There were statistically significant differences between the groups $(\mathrm{P}<0.05)$. Our study indicated that significantly different $\mathrm{HBsAg}$ positivity rates existed among the group of subjects 18 - 30 years old and those 30 years old or older $(\mathrm{P}<0.05)$. Furthermore, the infection rate among subjects 30 - 40 years old was the highest, followed by those in the age group of 40 - 50 years old, consistent with the findings of a previous epidemiological study (13). We believe that this trend could be attributed to the universal implementation of the vaccination program in China (14).

We also found statistically significant differences in HBsAg positivity in men versus women $(\mathrm{P}<0.05)$; similar results have been found in other studies $(15,16)$. We believe that possible explanations for this discrepancy include differences in lifestyle or behavior between men and women, such as drinking, smoking, extensive social ranges, sexual activity, etc. Also, for the men migrant workers, it is urgently to adjust their lifestyle.

The high prevalence of hepatitis $\mathrm{B}$ among migrant workers implies the need for the management of hepatitis B in these workers. Altogether, our study reflects the absence of a health policy for fighting this disease. About less than one-third of our study participants knew that chronic hepatitis B infection can lead to serious health problems such as liver failure, cirrhosis and liver cancer. However, we identified some important knowledge deficits, particularly with reference to routes of hepatitis B transmission. For example, less than $40 \%$ of migrant workers knew that hepatitis B can be transferred by sexual contact and from mother to infant. In addition, 32\% of migrant workers knew that it is necessary to receive a booster vaccination.

Thus, hepatitis B prevention and control among migrant workers are very important. Based on our findings, we suggest that effective programs need strengthening from the following aspects. First, increasing the coverage of hepatitis B vaccination could fully protect susceptible populations. Although hepatitis B can be prevalent in a population, it is preventable. Prevention is the only safe strategy against the high prevalence of the disease. Studies have demonstrated that no previous history of vaccination significantly increased the risk of the HBsAg infection (17). Hepatitis B vaccination was first conducted in China in 1992; its use was progressively expanded over the following years. After nearly two decades of effort, the HBsAg-positive rate in the general population of China dropped from 9.75\% in 1992 to 7.18\% in 2006 (18). This decrease illustrates the need for and importance of expanding and reintensifying immunization programs with extension to adults, especially migrant workers, who remain susceptible to infection. Of course, this fact means that the government should pay greater attention to the floating population in the expansion of the immune population. Second, more campaigns should aim to increase hepatitis B serologic testing rates, to control the source of infection and to reduce transmission among migrant workers. Hepatitis B testing allows for the identification of chronically infected individuals who could benefit from antiviral therapy and should take precautions to avoid infecting others, as well as susceptible individuals who should be vaccinated against the disease (19). Third, it is necessary to strengthen health education among migrant workers and improve their awareness of hepatitis B. Our study indicated a lack of health education campaigns targeting hepatitis B in migrant workers, which might also have contributed to this serious absence of knowledge. Having sufficient knowledge and proper attitudes toward hepatitis B are cornerstones of preventing the spread of the disease. Many studies have demonstrated that media campaigns can positively impact hepatitis B knowledge levels and preventive behaviors (20-22); however, hepatitis $B$ infection remains a great challenge for disease control in China. Therefore, more awareness raising and education campaigns among migrant workers are needed. According to our research results, the government needs to undertake more long-term measures. This need will require efforts from all walks of life.

Our study has several strengths: first, population-based sampling methods were used in this study, second, we administered the survey face-to-face and had a relatively good cooperation rate, and the third is its representativeness for one nation. However, several limitations to this study are also worth noting. First, the study was a popula- 
tion-based, cross-sectional survey, and the cross-sectional design hampered causality inference, and also some acute patients with hepatitis B cannot be tested. Second, the survey was subject to information and recall bias. Third, certain limitations of this pilot study are related to the small sample size and convenience sampling in a single city, as well as the difficulties inherent in sampling migrant populations. Although the study was conducted in a limited number of subjects, the outcomes interestingly revealed several findings about the prevalence of hepatitis B and knowledge regarding hepatitis B among migrant workers in Shandong Province, China. The outcomes could be useful in the design and implementation of future prevention strategies.

\section{Acknowledgements}

We heartily acknowledge the cooperation and support of the migrant workers who participated in our study.

\section{Authors' Contributions}

Yan Yang has full access to all of the data in the study and takes responsibility for the integrity of the data and the accuracy of the data analysis. Ming Yan participated in study concept and design. Wei Zhang, Jie Li, Shuqing Li participated in acquisition of data. Analysis and interpretation of data was done by Yan Yang, Meng Yue and Xiaohua Wang. Yan Yang contributed in drafting of the manuscript.

\section{Funding/Support}

This work was supported by Science and Technology Planning Project of Shandong Province, China (grant number 2007GG30002002). We declare that funders had no role in study design, data collection and analysis, decision to publish, or preparation of the manuscript.

\section{References}

1. Wu Z, Yao S. Intermigration and intramigration in China: A theoretical and empirical analysis. China Economic Review. 2003;14(4):371-85.

2. China National Bureau of Statistics (CNBS). Characteristics of Chinese Rural Migrants: 2000.Beijing; 2001.

3. National Population and Family Planning Commission.. [China Report on National Developments of Migrants in China in 2010].Beijing,China; 2010.

4. Liu J, Fan D. Hepatitis B in China. Lancet. 2007;369(9573):1582-3.
5. Lian JQ, Yang XF, Zhao RR, Zhao YY, Li Y, Zhang Y, et al. Expression profiles of circulating cytokines, chemokines and immune cells in patients with hepatitis B virus infection. Hepat Mon. 2014;14(6)

6. Hu Z, Yang J, Xiong G, Shi H, Yuan Y, Fan L, et al. HLA-DPB1 Variant Effect on Hepatitis B Virus Clearance and Liver Cirrhosis Development Among Southwest Chinese Population. Hepat Mon. $2014 ; 14(8)$.

7. Luo Z, Li L, Ruan B. Impact of the implementation of a vaccination strategy on hepatitis B virus infections in China over a 20-years period. Int J Infect Dis. 2012;16(2):e82-8.

8. Liang X, Bi S, Yang W, Wang L, Cui G, Cui F, et al. Reprint of: Epidemiological serosurvey of Hepatitis B in China-declining HBV prevalence due to Hepatitis B vaccination. Vaccine. 2013;31 Suppl 9:[21-8.

9. Wang Y, Jia J. Control of hepatitis B in China: prevention and treatment. Expert Rev Anti Infect Ther. 2011;9(1):21-5.

10. Juan LI. The Investigation and Analysis about Migrant Workers Infected by Hepatitis B Virus among 2462 Cases. Guide of China Medicine. 2011;32:017.

11. Centers for Disease Control and Prevention.. Interpretation of hepatitis B serologic test results. 2005. Available from: http://www. cdc.gov/hepatitis/HBV/PDFs/SerologicChartv8.pdf.

12. Zhang L, Xu A, Yan B, Song L, Li M, Xiao Z, et al. A significant reduction in hepatitis B virus infection among the children of Shandong Province, China: the effect of 15 years of universal infant hepatitis B vaccination. Int J Infect Dis. 2010;14(6):e483-8.

13. Xia G, Liu C, Cao H, Bi S, Zhan M, Su C, et al. Prevalence of hepatitis $B$ and $C$ virus infections in the general Chinese population. Results from a nationwide cross-sectional seroepidemiologic study of hepatitis A, B, C, D, and E virus infections in China, 1992. International Hepatology Communications. 1996;5(1):62-73.

14. Rezaee-Zavareh MS, Einollahi B. Hepatitis B vaccination: needs a revision. Hepat Mon. 2014;14(3).

15. Wang CS, Chang TT, Yao WJ, Chou P. Comparison of hepatitis B virus and hepatitis $C$ virus prevalence and risk factors in a community-based study. Am JTrop Med Hyg. 2002;66(4):389-93.

16. Chen P, Yu C, Ruan B, Yang S, Ren J, Xu W, et al. Prevalence of hepatitis B in insular regions of southeast China: a community-based study. PLoS One. 2013;8(2).

17. Zhang Y, Fang W, Fan L, Gao X, Guo Y, Huang W, et al. Hepatitis B surface antigen prevalence among 12,393 rural women of childbearing age in Hainan Province, China: a cross-sectional study. Virol J. 2013;10:25.

18. Liang X, Bi S, Yang W, Wang L, Cui G, Cui F, et al. Evaluation of the impact of hepatitis B vaccination among children born during 1992-2005 in China. JInfect Dis. 2009;200(1):39-47.

19. Jenkins CN, Buu C, Berger W, Son DT. Liver carcinoma prevention among Asian Pacific Islanders. Getting hepatitis B shots into arms. Cancer. 2001;91(1 Suppl):252-6.

20. McPhee SJ, Nguyen T, Euler GL, Mock J, Wong C, Lam T, et al. Successful promotion of hepatitis B vaccinations among Vietnamese-American children ages 3 to 18: results of a controlled trial. Pediatrics. 2003;111(6 Pt 1):1278-88.

21. Halpin HA, Morales-Suarez-Varela MM, Martin-Moreno JM Chronic disease prevention and the New Public Health. Public Health Reviews. 2010;32:120-54.

22. Taylor VM, Seng P, Acorda E, Sawn L, Li L. Hepatitis B knowledge and practices among Cambodian immigrants. J Cancer Educ. 2009;24(2):100-4. 